\title{
Multi-optical-wavelength ultrasound-modulated optical tomography: a phantom study
}

\author{
Chulhong Kim and Lihong V. Wang* \\ Department of Biomedical Engineering, Optical Imaging Laboratory, Washington University in St. Louis, \\ Campus Box 1097, One Brookings Drive, St. Louis, Missouri 63130, USA \\ *Corresponding author: lhwang@biomed.wustl.edu \\ Received April 12, 2007; revised July 2, 2007; accepted July 6, 2007; \\ posted July 9, 2007 (Doc. ID 82070); published August 2, 2007
}

\begin{abstract}
We used multiple optical wavelengths to study ultrasound-modulated optical tomography (UOT) in tissue phantoms. By using intense acoustic bursts and a CCD camera-based speckle contrast detection technique, we observed variations of the ultrasound-modulated signal at various optical absorptions. The experimental variations were found to be highly correlated with predictions from Monte Carlo simulations. By irradiating the sample at two optical wavelengths, we quantitatively estimated the total concentration and the concentration ratio of double dyes in objects embedded in tissue phantoms. The results suggest that UOT can potentially provide noninvasive functional imaging of the total concentration and oxygen saturation of hemoglobin in biological tissue. (C) 2007 Optical Society of America

OCIS codes: $030.1670,110.6150,110.7170,170.6480,170.3880$.
\end{abstract}

Imaging functional parameters of biological tissue, such as the total hemoglobin concentration $(\mathrm{HbT})$ and hemoglobin oxygen saturation $\left(\mathrm{SO}_{2}\right)$, is important in imaging brain activation [1] and studying tumor physiopathology [2]. Currently, diffuse optical tomography (DOT) [3] and photoacoustic imaging [4,5] can noninvasively estimate the $\mathrm{HbT}$ and $\mathrm{SO}_{2}$ in living subjects. However, pure optical imaging techniques, such as DOT, suffer from poor spatial resolution because of strong light scattering in tissue. Photoacoustic imaging overcomes this drawback of DOT because its spatial resolution is derived from ultrasound parameters. Similar to photoacoustic imaging, ultrasound-modulated optical tomography (UOT) [6] is capable of supplying strong optical contrast and high ultrasonic spatial resolution. Therefore, UOT has the potential for functional imaging based on optical contrast.

In UOT, multiply scattered light passing through an ultrasonic focal volume is acoustically phasemodulated by both ultrasound-induced particle displacement and changes in the refractive index. By computing the modulation depth-defined as the ratio of the ultrasound-modulated light intensity to the unmodulated light intensity - at each scanned ultrasonic focal volume, images representative of the optical properties of the tissue are formed. Leutz and Maret [7], Wang [8], and Sakadžić and Wang [9] have theoretically modeled the mechanisms of UOT. At present, a low signal-to-noise ratio (SNR) is the major challenge in UOT. A number of techniques have been developed to detect weak modulated signals efficiently [10-12]. However, rather than devoting efforts to the detection of weak signals, Kim et al. [13] and Zemp et al. [14] explored the use of intense acoustic bursts as a significant signal enhancement mechanism.

In this Letter, for the first time to the best of our knowledge, we performed UOT with two optical wavelengths as a means of studying the feasibility of functional imaging of tissue. By employing intense acoustic bursts and using the speckle contrast detection technique $[13,14]$ at two optical wavelengths, we quantitatively estimated the relative total concentration of red and blue dye mixtures, and the ratio of the red dye concentration to the total concentration in objects buried in tissue phantoms. In addition, the dependence of UOT signals on optical absorption was modeled with Monte Carlo simulations. The theoretical and experimental results were compared.

The experimental setup is illustrated in Fig. 1. Two lasers (JDS Uniphase, HeNe 1145: $633 \mathrm{~nm}$ wavelength, and Melles Griot, 56ICS153/HS: $657 \mathrm{~nm}$ wavelength) sequentially illuminated a tissue mimicking phantom. An average laser power of $\sim 30 \mathrm{~mW} / \mathrm{cm}^{2}$ was delivered to the sample. A narrowband high-power focused ultrasound transducer (Ultran, VHP100-1-138: $1 \mathrm{MHz}$ central frequency; 25 -mm active aperture; $38 \mathrm{~mm}$ focal length) generated acoustic waves. The ultrasonic focal zone was $\sim 2 \mathrm{~mm}$ in diameter and $\sim 20 \mathrm{~mm}$ in length. An ultrasound peak pressure of less than $1.9 \mathrm{MPa}$ was applied at the focal point. Therefore, the mechanical index-defined as the ultrasound peak pressure divided by the square root of the center frequency and utilized as a gauge of the mechanical bioeffects of diagnostic ultrasound on biological tissues-at this frequency was smaller than 1.9 , which is within the typical safety limit [15]. The duration of each acoustic burst was set to $2 \mathrm{~ms}$. We used a gelatincornstarch phantom (10\% gelatin and 10\% corn-

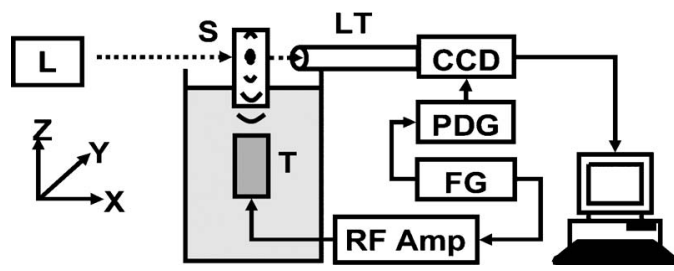

Fig. 1. Experimental setup: L, laser; CCD, CCD camera; RF amp, RF amplifier; FG, function generator; PDG, pulsedelay generator; T, ultrasound transducer; S, sample; LT, lens tube. 


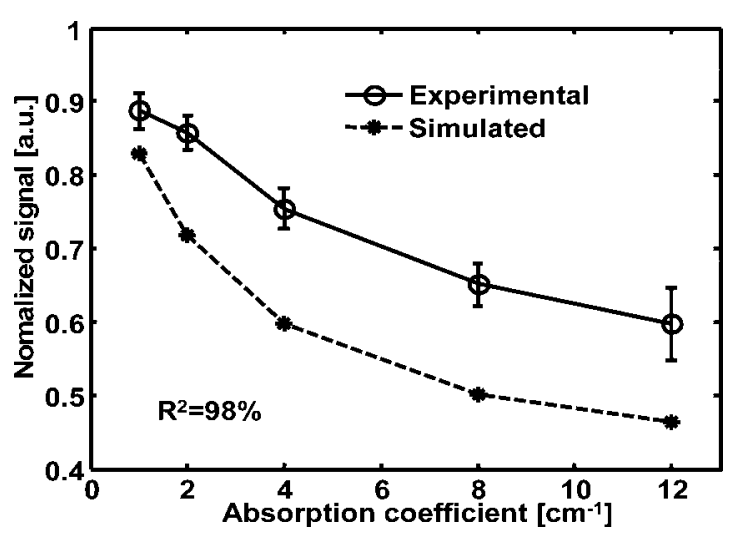

Fig. 2. Comparison of simulated modulation depths and experimentally measured changes in speckle contrast at various values of the optical absorption coefficient.

starch by weight) whose optical reduced scattering coefficient was $\sim 9 \mathrm{~cm}^{-1}$ at both wavelengths. A red dye was made from the mixture of Fiesta Red and India Red inks, whereas a blue dye was made of Trypan Blue. The absorption coefficients of the red dye in the mother liquid at 633 and $657 \mathrm{~nm}$ wavelengths were $\sim 2.2$ and $\sim 1.0 \mathrm{~cm}^{-1}$, respectively, whereas the absorption coefficients of the blue dye in the mother liquid at the two wavelengths were $\sim 11.9$ and $\sim 5.2 \mathrm{~cm}^{-1}$, respectively. We made optically absorptive objects by compounding the dye solutions with $10 \%$ gelatin. Speckle patterns emerging from the sample were captured by a CCD camera (Basler, A312f: 12 bit, $640 \times 480$ pixels). By placing a lens tube, which acted as an iris, in front of the CCD camera, we matched the average speckle size $D_{s}$ to a single CCD pixel size according to the relation $D_{s}=2.44 \lambda L / D_{i}$, where $\lambda$ is the light wavelength, $L$ is the distance of the lens tube, and $D_{i}$ is the aperture size of the lens tube. Ultrasonic tone bursts-synthesized by a function generator (Agilent, 33250A) and amplified by an $\mathrm{RF}$ amplifier (Amplifier Research, 75A250)—drove the ultrasound transducer. We used a low $1 \mathrm{~Hz}$ duty cycle to prevent damage to the transducer. The function generator triggered a pulse-delay generator (Stanford Research, DG535) that produced two CCD trigger pulses for each burst. An image was captured with an ultrasound burst; then, another one was captured without. The exposure time of the CCD camera was equivalent to the duration of an ultrasonic burst. The laser speckle contrast, defined as the ratio of the standard deviation to the mean intensity of the CCD image, was computed with and without ultrasound modulation. The UOT signal was defined as the change in speckle contrast between the ultrasound on and off conditions (off value-on value). We averaged 15 pairs of on-off measurements.

By irradiating the sample at two optical wavelengths, $\lambda_{1}$ and $\lambda_{2}$, independently, we obtained two UOT images that represent the distributions of light fluences at those wavelengths. The UOT signal acquired from each ultrasonically scanned sample volume was normalized by the average UOT signal from the background outside the buried objects. We approximated the dependence of the amplitude of the UOT signal on the local optical absorption to
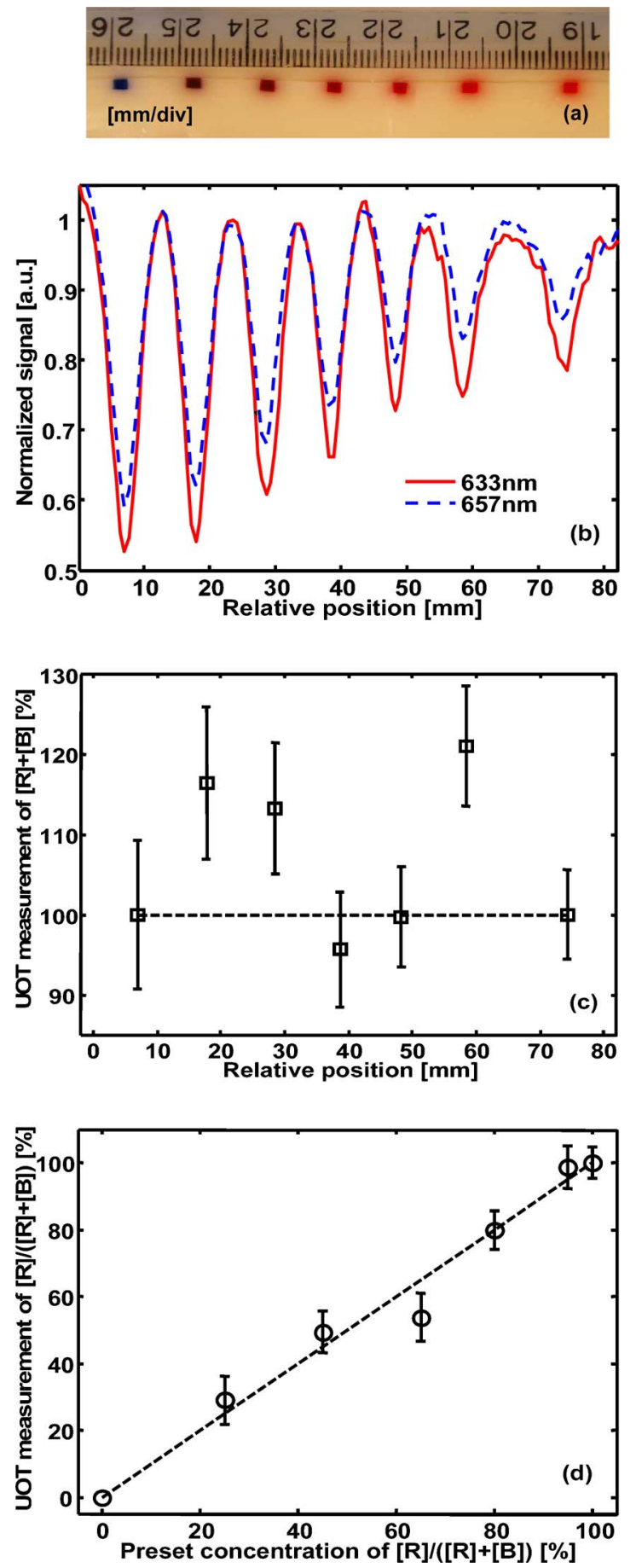

Fig. 3. (Color online) (a) Photograph of the tissue mimicking the phantom, containing seven objects dyed with different ratios of red dye concentration, $[R]$, to the total (red and blue) dye concentrations, $[R]+[B]$. (b) One-dimensional images of the phantom at wavelengths of 633 and $657 \mathrm{~nm}$. (c) UOT measurements of the total dye concentration, $[R]$ $+[B]$, and $(d)$ fraction of the red dye concentration in the total dye concentration, $[R] /([R]+[B])$.

$\exp \left(-\mu_{a} d\right)$, where $\mu_{a}$ is the optical absorption coefficient and $d$ is the diameter of an embedded object. This dependence was used to calculate the concentrations of both dyes. Therefore, the total dye concentration, $[R]+[B]$, and the ratio of the red dye concentra- 
tion to the total dye concentration, $[R] /([R]+[B])$, can be calculated as follows:

$$
\begin{gathered}
{[R]+[B]=\frac{\mu_{a}\left(\lambda_{1}\right) \Delta \varepsilon\left(\lambda_{2}\right)-\mu_{a}\left(\lambda_{2}\right) \Delta \varepsilon\left(\lambda_{1}\right)}{\varepsilon_{B}\left(\lambda_{1}\right) \varepsilon_{R}\left(\lambda_{2}\right)-\varepsilon_{B}\left(\lambda_{2}\right) \varepsilon_{R}\left(\lambda_{1}\right)},} \\
\frac{[R]}{[R]+[B]}=\frac{\mu_{a}\left(\lambda_{2}\right) \varepsilon_{B}\left(\lambda_{1}\right)-\mu_{a}\left(\lambda_{1}\right) \varepsilon_{B}\left(\lambda_{2}\right)}{\mu_{a}\left(\lambda_{1}\right) \Delta \varepsilon\left(\lambda_{2}\right)-\mu_{a}\left(\lambda_{2}\right) \Delta \varepsilon\left(\lambda_{1}\right)},
\end{gathered}
$$

where $[R]$ and $[B]$ are the concentrations of red and blue dyes, respectively, relative to their mother liquids; $\mu_{a}$ is the measured relative optical absorption; $\varepsilon_{R}$ and $\varepsilon_{B}$ are the absorption coefficients of pure red and blue mother liquids, respectively, measured with our UOT system; and $\Delta \varepsilon=\varepsilon_{R}-\varepsilon_{B}$.

We computed modulation depths using Monte Carlo simulations at different optical absorptions and compared the simulated values to the experimental results (Fig. 2). The Monte Carlo simulations are based on the temporal correlation transfer equation for acoustically modulated multiply scattered light [9]. Using the numerical method, we can map the distribution of ultrasound-modulated light intensity in isotropic optical scattering medium (scattering anisotropy: 0) with heterogeneous optical properties and a nonuniform ultrasonic field [9]. The simulation parameters included a cylindrical-ultrasonic-focal zone of $2 \mathrm{~mm}$ in diameter and $20 \mathrm{~mm}$ in length, an ultrasonic pressure of $1.5 \mathrm{MPa}$, an optical wavelength of $633 \mathrm{~nm}$, a $20 \mathrm{~mm}$ thick isotropic scattering medium with a reduced scattering coefficient of $\sim 9 \mathrm{~cm}^{-1}$, and a cylindrical-optical-absorbing object of $2.2 \mathrm{~mm}$ in diameter and $11 \mathrm{~mm}$ in length. The optical absorption coefficient of the embedded object in the medium varied from 1 to $12 \mathrm{~cm}^{-1}$. As the absorption coefficient increases, the modulation depth decreases as shown in Fig. 2. The decay profile is close to an exponential fit instead of a linear fit. To validate our simulation results, we scanned a $20 \mathrm{~mm}$ thick phantom containing five Trypan-Blue-dyed objects $(2.2 \mathrm{~mm} \times 2.2 \mathrm{~mm} \times 11 \mathrm{~mm}$ along the $X, Y$, and $Z$ axes, respectively). We used the same experimental parameters as we did in the above simulation. Although they have different absolute values, the experimental and simulated results are highly correlated ( $R^{2}$ value: $\left.98 \%\right)$ as shown in Fig. 2.

To investigate the potential of measuring $\mathrm{HbT}$ and $\mathrm{SO}_{2}$ using UOT, we scanned a phantom at two optical wavelengths (633 and $657 \mathrm{~nm}$ ). An acoustic peak pressure of $1 \mathrm{MPa}$ was applied to the sample. The phantom was $20 \mathrm{~mm}$ thick and contained seven objects [Fig. 3(a)]. The sizes of all seven targets were identical: $2.2 \mathrm{~mm} \times 2.2 \mathrm{~mm} \times 11 \mathrm{~mm}$. Two dye solutions were mixed at seven concentration ratios $([R]:[B]=0: 100,25: 75,45: 55,65: 35,80: 20,95: 5$, and 100:0 [\%]) to mimic different levels of $\mathrm{SO}_{2}$. The rela- tive positions in Figs. 3(b) and 3(c) indicate a horizontal axis across all targets in Fig. 3(a). Figure 3(b) shows 1D images obtained at the two optical wavelengths. By substituting the relative optical absorption coefficients measured from the 1D images into Eqs. (1) and (2), we estimated the values of $[R]+[B]$ [Fig. 3(c)] and $[R] /([R]+[B])[$ Fig. 3(d)]. The experimentally quantified total concentration $[R]+[B]$ in each of the seven objects is close to the preset value of $100 \%$ with an error up to $\sim 20 \%$. The estimated $[R] /([R]+[B])([R]+[B])$ values match the actual preset concentrations well with an $R^{2}$ value of $99 \%$. The spatial resolution, defined as the one-way distance between the $25 \%$ and $75 \%$ points across the maximum and minimum of the edge spread function, is $\sim 2 \mathrm{~mm}$ at both wavelengths, which is comparable with the ultrasonic beam size. The SNRs, defined as the ratio of the mean to the standard deviation of the UOT signals, are $110 \pm 17$ (standard error) and $213 \pm 9$ at the wavelengths of 633 and $657 \mathrm{~nm}$, respectively.

In conclusion, this research has demonstrated the feasibility of functional UOT in tissue phantoms. The estimated total dye concentration and the ratio of the red dye concentration to the total dye concentration match the actual preset values well. The technique can potentially be extended to monitoring total $\mathrm{HbT}$ and measuring absolute $\mathrm{SO}_{2}$ in vivo.

We thank H. F. Zhang and R. J. Zemp for fruitful scientific discussions. This research was supported by NIH grants R33 CA 094267 and R01 CA106728.

\section{References}

1. I. Vanzetta and A. Grinvald, Science 286, 1555 (1999),

2. C. Menon and D. L. Frake, Cancer Lett. 221, 225 (2005)

3. J. P. Culver, A. M. Siegel, J. J. Stott, and D. A. Boas, Opt. Lett. 28, 2016 (2003).

4. X. Wang, Y. Pang, G. Ku, X. Xie, G. Stoica, and L.-H. Wang, Nat. Biotechnol. 21, 803 (2003).

5. H. F. Zhang, K. Maslov, G. Stoica, and L.-H. Wang, Nat. Biotechnol. 24, 848 (2006).

6. L.-H. Wang, S. L. Jacques, and X. Zhao, Opt. Lett. 20, 629 (1995).

7. W. Leutz and G. Maret, Physica B 204, 14 (1995).

8. L.-H. V. Wang, Phys. Rev. Lett. 87, 043903 (2001).

9. S. Sakadžić and L.-H. V. Wang, Phys. Rev. E 74, 036618 (2006).

10. S. Leveque, A. C. Boccara, M. Lebec, and H. SaintJalmes, Opt. Lett. 24, 181 (1999).

11. S. Sakadžić and L.-H. V. Wang, Opt. Lett. 29, 2770 (2004).

12. T. W. Murray, L. Sui, G. Maguluri, R. A. Roy, A. Nieva, F. Blonigen, and C. A. DiMarzio, Opt. Lett. 29, 2509 (2004).

13. C. Kim, R. J. Zemp, and L.-H. V. Wang, Opt. Lett. 31, 2423 (2006).

14. R. J. Zemp, S. Sakadžić, and L.-H. V. Wang, Phys. Rev. E 73, 061920 (2006).

15. D. Dalecki, Biomed. Eng. (NY) 6, 18.1 (2004). 